\title{
New Guidance Laws and Autopilot Designs for Intercepting Missiles with TVC and DCS
}

\author{
Chia-Hao Cheng, Fu-Kuang Yeh and Li-Chen Fu, IEEE Fellow
}

\begin{abstract}
In this paper, guidance law (GL) and autopilot system, the decision parts of an airframe, are discussed, and specially the intercepting missiles are the plants considered. For a non-aerodynamically maneuvering airframe, a wingless structure based on thrust vector control (TVC) and divert control system (DCS) is used. To design a robust autopilot system, the sliding mode theory is applied. Besides, two new missile GLs are presented, which utilize the optimal control theory, inverse dynamics technique, and Lyapunov analysis. In addition, stability of the integrated guidance/autopilot (G/A) system and the design methodology of the system parameters are discussed. Finally, various simulations including aerodynamic model are demonstrated to examine the feasibility and performance of the missile's integrated G/A systems.
\end{abstract}

\section{INTRODUCTION}

The control of flight vehicles has been an important topic ever since the range of human activities was extended from the earth to the universe. It becomes even more influential to mankind as the modern technology progresses, such as invention of the missiles that protect our homeland from a disaster. In this paper, the controller design of defense missiles are discussed. Specially, GLs and autopilot systems are the two in which we are interested among all control units. Regarding the GLs, proportional navigation has been implemented on intercepting missiles for half a century, but it and its derivations from optimal theory are still essential research topics and are discussed systematically in [2] and [3]. Nevertheless, many of other solutions are excellent as well and have been proposed, such as differential geometric approach [5], the game theory, the fuzzy method, etc. Conventionally, the missile autopilot system designs are based on the classical three-loop autopilot design [2]. However, the missile autopilot systems with quaternion representation and sliding mode control design in our previous research works [6] and [7] have acquired excellent performance, and are reinvented in this paper.

In this paper, two new GLs and one autopilot system are proposed. Both the missile GLs involve the optimal control theory, inverse dynamics technique, and Lyapunov analysis in two different mathematical models, and the comparisons between their characteristics and performances are also made. To design a whole-phase-single-strategy autopilot system that is capable of maneuvering in both endo- and exo-atmosphere, we apply the sliding mode theory and use a

\footnotetext{
Chia-Hao Cheng and Fu-Kuang Yeh are with the Department of Electrical Engineering, National Taiwan University, Taipei, Taiwan, R.O.C. r91921009@ntu. edu. tw

Li-Chen Fu is with the College of Electrical Engineering and Computer Science, National Taiwan University, Taipei, Taiwan, R.O.C. lichen@ntu.edu.tw
}

wingless missile airframe based on TVC/DCS. In addition to the respective GLs and autopilot subsystems, the integrated G/A systems are discussed, and the design methodologies of the system parameters are suggested. Various simulations including aerodynamic model are demonstrated at the last to verify the feasibility and test the performance of the missile's integrated G/A systems.

\section{AUTOPILOT DESIGN WITH TVC AND DCS}

Generally speaking, GLs and autopilot systems are both the strategic parts in an airframe; decisions and control commands are made by them. GLs guide the airframe's directions based on the current state of the airframe and the circumstances, while autopilot systems which depend on a well-designed controller follow the guidance commands stably. The illustrative and conceptual flowchart of the commands in an airframe is shown in Fig. 1, and the autopilot systems are discussed in this section. An autopilot system is a mechanism which receives the acceleration commands from GLs and controls the actuators to stabilize the airframe while performing those commands. In this paper, the autopilot system is divided into three parts for design, but the actuator dynamics is excluded from our topics.

Fig. 2 shows the architecture of the missile, where TVC is a mechanism realized by a moveable tail nozzle, and DCS is an additional auxiliary propellent system mounted on lateral of the missile. Both TVC and DCS have the advantage of maneuvering ability in both endo- and exo-atmosphere, whereas control surfaces work only in endoatmosphere. With this reason and a motivation to design a whole-phasesingle-strategy controller, we choose an airframe without control surface, which dominates the aerodynamic force. By this, the designed whole-phase-single-strategy controller will be more compatible with the circumstance where less

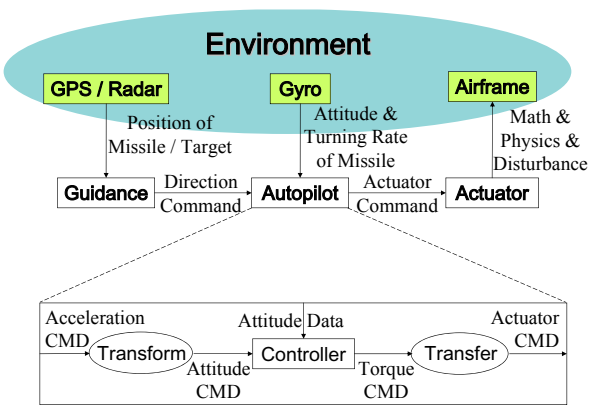

Fig. 1. Subsystems of an Airframe 


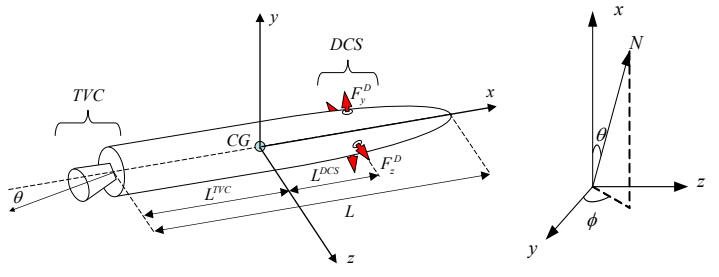

Fig. 2. (a) Missile Configuration (b) TVC Moving Angles

aerodynamic influence on the airframe in both endo- and exo-atmosphere is expected. Based on physics laws, the kinematic equations of the airframe are composed of those of both TVC and DCS, that is

1) Translational Kinematics:

$$
\vec{F}_{b}^{\text {prop }}=\vec{F}_{b}^{T V C}+\vec{F}_{b}^{D C S}=\left[\begin{array}{c}
N \cos \theta \\
N \sin \theta \cos \phi+F_{y}^{D} \\
N \sin \theta \sin \phi+F_{z}^{D}
\end{array}\right]
$$

\section{2) Rotational Kinematics:}

$$
\begin{aligned}
\vec{\tau}_{b}^{\text {prop }} & =\vec{\tau}_{b}^{T V C}+\vec{\tau}_{b}^{D C S} \\
& =\left[\begin{array}{c}
N l^{T V C} \sin \theta \sin \phi-l^{D C S} F_{z}^{D} \\
-N l^{T V C} \sin \theta \cos \phi+l^{D C S} F_{y}^{D}
\end{array}\right]
\end{aligned}
$$

\section{A. Translate Torque CMDs into Actuator CMDs}

The "Transfer" part in Fig. 1 is designed to calculate the actuator commands that can reach the desired torque commands. It is interesting to note that a TVC system generates an unpleasantly accompanied lateral force while it is trying to generate a rotational torque by moving the nozzle. This accompaniment, however, can be easily eliminated by the addition of the auxiliary DCS. In other words, the cooperative strategy between TVC and DCS ensures a rotational torque without lateral force accompanied, which is a proper way for an airframe to change the direction of heading. To do so, we simply let the summation of the last two terms of (1) be zero, i.e.,

$$
F_{y}^{D}=-N \sin \theta \cos \phi \quad ; \quad F_{z}^{D}=-N \sin \theta \sin \phi,
$$

and thus get the desired relationship between TVC moving angles and the required DCS propulsion. Substituting them into (2), we have:

$$
\begin{aligned}
\tau_{b y} & =\left(N l^{T V C}+N l^{D C S}\right) \sin \theta \sin \phi \\
\tau_{b z} & =-\left(N l^{T V C}+N l^{D C S}\right) \sin \theta \cos \phi,
\end{aligned}
$$

which are the rotational torque generated by the cooperation of the actuators TVC and DCS. To attain the desired torque commands $\tau_{D}$, the required actuator commands by reformulating (4) and (5) should be:

$$
\begin{gathered}
\sin \theta_{D}=\frac{\sqrt{\tau_{D b y}^{2}+\tau_{D b}^{2}}}{N l^{T V C}+N l^{D C S}} \\
\sin \phi_{D}=\frac{\tau_{D b y}}{\sqrt{\tau_{D b y}^{2}+\tau_{D b z}^{2}}} ; \quad \cos \phi_{D}=\frac{-\tau_{D b z}}{\sqrt{\tau_{D b y}^{2}+\tau_{D b z}^{2}}}
\end{gathered}
$$

Substituting these required TVC moving angles into (3), we can obtain the correspondingly required DCS propulsion:

$$
F_{y}^{D}=\frac{\tau_{D b z}}{l^{T V C}+l^{D C S}} \quad ; \quad F_{z}^{D}=\frac{-\tau_{D b y}}{l^{T V C}+l^{D C S}}
$$

\section{B. Translate Attitude CMDs into Torque CMDs}

The "Controller" part in Fig. 1 generated the torque commands for the desired attitude commands. The attitude is expressed by quaternion $q$ and angular velocity $\vec{\omega} ;\left(q_{e}, \vec{\omega}_{e}\right)$ are defined as the errors between desired values $\left(q_{d}, \vec{\omega}_{d}\right)$ and current values $(q, \vec{\omega})$ as follows:

$$
q_{e}=\left[\begin{array}{l}
q_{e 1} \\
q_{e 2} \\
q_{e 3} \\
q_{e 4}
\end{array}\right]=\left[\begin{array}{rrrr}
q_{d 4} & q_{d 3} & -q_{d 2} & -q_{d 1} \\
-q_{d 3} & q_{d 4} & q_{d 1} & -q_{d 2} \\
q_{d 2} & -q_{d 1} & q_{d 4} & -q_{d 3} \\
q_{d 1} & q_{d 2} & q_{d 3} & q_{d 4}
\end{array}\right]\left[\begin{array}{c}
q_{1} \\
q_{2} \\
q_{3} \\
q_{4}
\end{array}\right]
$$

where the quaternion is defined as:

$$
q^{(4 \times 1)}=\left[\begin{array}{c}
\vec{q}^{(3 \times 1)} \\
q_{4}
\end{array}\right]=\left[\begin{array}{llll}
q_{1} & q_{2} & q_{3} & q_{4}
\end{array}\right]^{T}=\left[\begin{array}{c}
\hat{e} \sin (\phi / 2) \\
\cos (\phi / 2)
\end{array}\right],
$$

where the unit vector $\hat{e}=\left[\begin{array}{lll}e_{1} & e_{2} & e_{3}\end{array}\right]^{T}$ and angle $\phi$ are the Euler Axis and Angle exactly. To generate the torque commands that eliminate the attitude errors $q_{e}$ and $\vec{\omega}_{e}$, a controller design based on sliding mode control theory is proposed. Define the sliding surface variable as

$$
S_{a}=P \vec{q}_{e}+\vec{\omega}_{e},
$$

where $P$ is a $3 \times 3$ P.D. matrix, whereas $S_{a}, \vec{q}_{e}$, and $\vec{\omega}_{e}$ are $3 \times 1$ vectors. Choose the Lyapunov function candidate as

$$
V_{a}\left(S_{a}\right)=\frac{1}{2} S_{a}^{T} J S_{a},
$$

where $J$ is the inertia matrix of the airframe. Taking the time derivative of $V_{a}$, we have:

$$
\dot{V}_{a}=S_{a}^{T}\left(\frac{1}{2} \dot{J} S_{a}+J P \dot{\vec{q}}_{e}+J \dot{\vec{\omega}}-J \dot{\vec{\omega}}_{d}\right)
$$

Now applying the kinematic equation of quaternion

$$
\dot{\vec{q}}_{e}=\frac{1}{2} \vec{q}_{e} \times \vec{\omega}_{e}+\frac{1}{2} q_{e 4} \vec{\omega}_{e}
$$

and the Euler's equation

$$
J \dot{\vec{\omega}}=\vec{\tau}_{b}-\dot{J} \vec{\omega}-\vec{\omega} \times(J \vec{\omega}),
$$

we then have:

$$
\begin{aligned}
\dot{V}_{a}= & S_{a}^{T}\left(\frac{1}{2} \dot{J} S_{a}+J P\left(\frac{1}{2} \vec{q}_{e} \times \vec{\omega}_{e}+\frac{1}{2} q_{e 4} \vec{\omega}_{e}\right)\right. \\
& \left.+\vec{\tau}_{b}-\dot{J} \vec{\omega}-\vec{\omega} \times(J \vec{\omega})-J \dot{\vec{\omega}}_{d}+d_{a}\right),
\end{aligned}
$$

where the torque command $\vec{\tau}_{b}$ is the control input of this error tracking system, and the disturbance $d_{a}^{(3 \times 1)}$ is specially taken into consideration, which comes from both parametric uncertainties and unmodelled dynamics involving aerodynamics, actuator dynamics, parasitic dynamics, etc. Without loss of generality, we assume that there exists 
an upper bound vector $d_{a}^{\max }$ on the disturbance $d_{a}$, i.e. $\left|d_{a i}\right| \leq d_{a i}^{\max }, i=1,2,3$. Design the control law as:

$$
\begin{aligned}
\vec{\tau}_{D b} & =-\frac{1}{2} \dot{J} S_{a}-J P\left(\frac{1}{2} \vec{q}_{e} \times \vec{\omega}_{e}+\frac{1}{2} q_{e} \vec{\omega}_{e}\right)+\dot{J} \vec{\omega} \\
& +\vec{\omega} \times(J \vec{\omega})+J \dot{\vec{\omega}}_{d}-\left(d_{a}^{\text {max }}+\eta_{a}\right) \operatorname{sgn}\left(S_{a}\right),
\end{aligned}
$$

where $\eta_{a}^{(3 \times 1)}>0$ is an adjustable vector variable, and $\operatorname{sgn}\left(S_{a}\right)=\left[\operatorname{sgn}\left(S_{a 1}\right), \operatorname{sgn}\left(S_{a 2}\right), \operatorname{sgn}\left(S_{a 3}\right)\right]^{T}$. With the designed control law, we get:

$\dot{V}_{a}=S_{a}^{T} d_{a}-S_{a}^{T} d_{a}^{\max } \operatorname{sgn}\left(S_{a}\right)-S_{a}^{T} \eta_{a} \operatorname{sgn}\left(S_{a}\right) \leq-\eta_{a}^{T}\left|S_{a}\right|$

Accordingly, the system trajectories will reach the sliding surface $S_{a}=0$ in finite time less than $\max _{i} \frac{\left|S_{a i}\left(t_{0}\right)\right|}{\eta_{a}}$, remain on it, and then are constrained by the sliding surface itself, namely

$$
S_{a}=P \vec{q}_{e}+\vec{\omega}_{e}=0
$$

Now, we shall verify that $\vec{\omega}_{e}$ and $\vec{q}_{e}$ will converge to zero when the above condition is reached. Define the Lyapunov function candidate as

$$
V_{q}\left(\vec{q}_{e}\right)=\vec{q}_{e}^{T} \vec{q}_{e}
$$

Take the time derivative of $V_{q}$ and combine with (18):

$\dot{V}_{q}=2 \vec{q}_{e}^{T}\left(\frac{1}{2} \vec{q}_{e} \times \vec{\omega}_{e}+\frac{1}{2} q_{e 4} \vec{\omega}_{e}\right)=q_{e 4} \vec{q}_{e}^{T} \vec{\omega}_{e}=-q_{e 4} \vec{q}_{e}^{T} P \vec{q}_{e}$

Substitute (18) into the kinematic equation of quaternion:

$$
\dot{q}_{e 4}=-\frac{1}{2} \vec{q}_{e} \cdot \vec{\omega}_{e}=\frac{1}{2} \vec{q}_{e}^{T} P \vec{q}_{e} \geq 0,
$$

which implies that $q_{e}$ is a non-decreasing positive variable. Define the domain of rotation angle $\phi$ to be between $-\pi$ and $\pi$, which implies a non-negative $q_{e 4}$, to avoid the sign ambiguity in the representation of quaternion. Thus $q_{e 4}=\cos \left(\frac{\phi}{2}\right) \geq 0$. If we select a nonzero $q_{e 4}\left(t_{0}\right)$, which is determined by the designable initial conditions, we have $0<q_{e 4}\left(t_{0}\right) \leq q_{e 4}(t), \forall t \geq 0$. Substituting it into (20), we have

$$
\dot{V}_{q} \leq-q_{e 4}\left(t_{0}\right) \vec{q}_{e}^{T} P \vec{q}_{e}<0,
$$

i.e. $V_{q}\left(\vec{q}_{e}\right)$ is P.D., and $\dot{V}_{q}\left(\vec{q}_{e}\right)$ is N.D. In addition, $V_{q}\left(\vec{q}_{e}\right) \rightarrow$ $\infty$ as $\left|\vec{q}_{e}\right| \rightarrow \infty$. According to the Lyapunov direct method, the equilibrium point $\vec{q}_{e}=0$ is globally asymptotically stable. Combined with (18), the asymptotical stability of $\vec{q}_{e}=0$ always implies the asymptotical stability of $\vec{\omega}_{e}$.

In summary, with the control law (16) the system trajectories will reach the sliding surface (18) in finite time less than $\max _{i} \frac{\left|S_{a i}\left(t_{0}\right)\right|}{\eta_{a}}$, remain on it, and it follows the asymptotic convergence of the attitude error $\vec{q}_{e}$ and the angular velocity error $\vec{\omega}_{e}$.

\section{Translate Acceleration CMDs into Attitude CMDs}

The "Transform" part in Fig. 1 transforms the acceleration commands from a GL into the compatible attitude commands in quaternion representation. In Fig. 3, the vector of the desired acceleration command $\vec{a}_{d}$ generated from the constant main propellant TVC is given by GLs, and the directions of $y_{b}$ and $z_{b}$ are the "don't care" factors for this

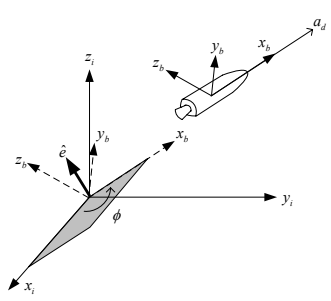

Fig. 3. Desire Quaternion

cylindrical, non-aerodynamically maneuvering airframe. To interpret the attitude commands in quaternion representation, we firstly set a reference coordinate frame, and then find the relationship between the reference coordinate frame and the acceleration command vector. According to the Euler's rotation theory, there exists a vector $\hat{e}$ and an angle $\phi$ such that $x_{i}$ will coincide with $x_{b}$ after undergoing an rotation of the angle $\phi$ by the vector $\hat{e}$. The vector $\hat{e}$ and the angle $\phi$ can be obtained by:

$$
\begin{aligned}
\hat{e} & =\frac{x_{i} \times x_{b}}{\left|x_{i} \times x_{b}\right|}=\frac{x_{i} \times a_{d}}{\left|x_{i} \times a_{d}\right|} \\
\phi & =\cos ^{-1}\left(\frac{x_{i} \cdot x_{b}}{\left|x_{i}\right|\left|x_{b}\right|}\right)=\cos ^{-1}\left(\frac{x_{i} \cdot a_{d}}{\left|x_{i}\right|\left|a_{d}\right|}\right),
\end{aligned}
$$

which can be used to find the desired quaternion from (9), and in turn yield the desired angular velocity by the kinematic equation of quaternion:

$$
\vec{\omega}_{d}=2\left[\begin{array}{rrrr}
q_{d 4} & q_{d 3} & -q_{d 2} & -q_{d 1} \\
-q_{d 3} & q_{d 4} & q_{d 1} & -q_{d 2} \\
q_{d 2} & -q_{d 1} & q_{d 4} & -q_{d 3}
\end{array}\right] \dot{q}_{d}
$$

\section{TWO NEW GUIDANCE LAWS AND ANALYSIS OF INTEGRATED G/A SYSTEMS}

In this section, two new GLs for intercepting missiles are proposed, and the integrated analysis for the integrated G/A systems are also discussed.

\section{A. Two 3-D Geometry Modelling}

In this subsection, the general missile intercepting problem is discussed, and two mathematical models are derived in terms of different viewpoints. Define the relative position, velocity, and acceleration to be the vectors from missile to target as follows:

$$
\vec{a}=\dot{\vec{v}}=\ddot{\vec{r}}=\ddot{\vec{r}}_{\text {Target }}-\ddot{\vec{r}}_{\text {Missile }},
$$

where $\vec{a}_{\text {Target }}$ and $\vec{a}_{\text {Missile }}$ are the acceleration generating from their own thrust regardless of gravity because of that both missile and target are affected by the same gravity (in this paper, we assume the earth is flat and gravity is constant). Note that $\vec{a}, \vec{v}$, and $\vec{r}$ are all in 3-D space, and thus they are generally not on the same plane. Knowing that any two vectors in 3-D space always form a plane, we let $\vec{r}$ and $\vec{v}$ be the two vectors to form a $\vec{r}-\vec{v}$ plane as shown in Fig. 4. Define $\hat{r}=\frac{\vec{r}}{|\vec{r}|}$, then 


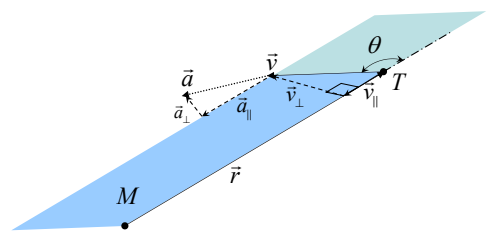

Fig. 4. 3-D Intercepting Model and the $\vec{r}-\vec{v}$ Plane

$$
\begin{aligned}
\frac{d}{d t}|\vec{r}| & =\frac{d}{d t}\left(\sqrt{r_{1}^{2}+r_{2}^{2}+r_{3}^{2}}\right)=\frac{\vec{r} \cdot \vec{v}}{|\vec{r}|} \\
\frac{d}{d t}|\vec{v}| & =\frac{d}{d t}\left(\sqrt{v_{1}^{2}+v_{2}^{2}+v_{3}^{2}}\right)=\frac{\vec{v} \cdot \vec{a}}{|\vec{v}|} \\
\frac{d}{d t} \hat{r} & =\frac{d}{d t}\left(\frac{\vec{r}}{|\vec{r}|}\right)=\frac{1}{|\vec{r}|} \vec{v}-\frac{\vec{v} \cdot \hat{r}}{|\vec{r}|} \hat{r}
\end{aligned}
$$

Referring to Fig. 4, it is obvious that the perfect interception can be guaranteed if $\vec{v}$ and $\vec{r}$ keep coincided with each other oppositely. Instead of this exactly coincident condition that is hardly met, however, the stable convergence of the relationship between $\vec{v}$ and $\vec{r}$ can also guarantee the intercepting requirements. Explicitly, the stably monotonic decreasing of $(\pi-\theta)$ and $\vec{v}_{\perp}$, the perpendicular component of $\vec{v}$ with respect to $\vec{r}$, can both guarantee successful interceptions. The two conditions, which are actually equivalent, are merely based on different viewpoints: angle and velocity. To meet the two intercepting conditions, dynamic equations of both are derived individually.

To begin with the viewpoint from angle, we proceed from the triangle equation $\vec{r} \cdot \vec{v}=|\vec{r}||\vec{v}| \cos \theta$. Differentiate it on both sides and make rearrangement to find $\dot{\theta}$ :

$$
\begin{aligned}
\dot{\theta} & =\frac{-|\vec{v}|}{|\vec{r}| \sin \theta}-\frac{\vec{r} \cdot \vec{a}}{|\vec{r}||\vec{v}| \sin \theta}+\frac{\vec{r} \cdot \vec{v} \cos \theta}{|\vec{r}|^{2} \sin \theta}+\frac{\vec{v} \cdot \vec{a} \cos \theta}{|\vec{v}|^{2} \sin \theta} \\
& =\frac{-|\vec{v}|}{|\vec{r}| \sin \theta}-\frac{\vec{r} \cdot \vec{a} \cos \theta}{\vec{r} \cdot \vec{v} \sin \theta}+\frac{|\vec{r}||\vec{v}| \cos ^{2} \theta}{|\vec{r}|^{2} \sin \theta}+\frac{\vec{v} \cdot \vec{a} \cos \theta}{|\vec{v}|^{2} \sin \theta} \\
& =-\frac{|\vec{v}|}{|\vec{r}|} \sin \theta+\left(\frac{\vec{v} \cdot \vec{a}}{\vec{v} \cdot \vec{v}}-\frac{\vec{r} \cdot \vec{a}}{\vec{r} \cdot \vec{v}}\right) \frac{\cos \theta}{\sin \theta}
\end{aligned}
$$

Define $\vec{a}=\vec{a}_{p}+\vec{a}_{n}+\vec{a}_{o}$, where $\vec{a}_{p}$ is the projection of $\vec{a}$ in the direction parallel to $\vec{v}, \vec{a}_{n}$ is the component of $\vec{a}$ on the $\vec{r}-\vec{v}$ plane and normal to the direction of $\vec{v}$, and

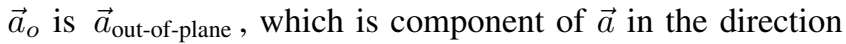
normal to the $\vec{r}-\vec{v}$ plane. Accordingly, we can find the following relationship:

$$
\begin{array}{ll}
\vec{v} \cdot \vec{a}_{o}=0 & \vec{r} \cdot \vec{a}_{o}=0 \\
\vec{v} \cdot \vec{a}_{p}=|\vec{v}|\left|\vec{a}_{p}\right| & \vec{r} \cdot \vec{a}_{p}=|\vec{r}|\left|\vec{a}_{p}\right| \cos \theta \\
\vec{v} \cdot \vec{a}_{n}=0 & \vec{r} \cdot \vec{a}_{n}=|\vec{r}|\left|\vec{a}_{n}\right| \cos \left(\theta \pm \frac{\pi}{2}\right)
\end{array}
$$

where \pm depends on which side of $\vec{a}$ the vector $\vec{v}$ is located. Thus (30) becomes:

$$
\dot{\theta}=-\frac{|\vec{v}|}{|\vec{r}|} \sin \theta-\frac{\left|\vec{a}_{n}\right|}{|\vec{v}|} \frac{\cos \left(\theta \pm \frac{\pi}{2}\right)}{\sin \theta}
$$

To discuss the sign, we can find out from Fig. 5 that if $\vec{a}_{n}$ is in the same direction with $((\vec{r} \times \vec{v}) \times \vec{v})$, then $\vec{r} \cdot \vec{a}_{n}=$ $|\vec{r}|\left|\vec{a}_{n}\right| \cos \left(\theta+\frac{\pi}{2}\right)=-|\vec{r}|\left|\vec{a}_{n}\right| \sin \theta$, and vice versa. Thus

$$
\dot{\theta}=-\frac{|\vec{v}|}{|\vec{r}|} \sin \theta+\operatorname{sgn}\left[((\vec{r} \times \vec{v}) \times \vec{v}) \cdot \vec{a}_{n}\right] \frac{\left|\vec{a}_{n}\right|}{|\vec{v}|}
$$

Let $\phi=\pi-\theta$ and reformulate the above equation, thus:

$$
\dot{\phi}=\frac{|\vec{v}|}{|\vec{r}|} \sin \phi-\operatorname{sgn}\left[((\vec{r} \times \vec{v}) \times \vec{v}) \cdot \vec{a}_{n}\right] \frac{\left|\vec{a}_{n}\right|}{|\vec{v}|}
$$

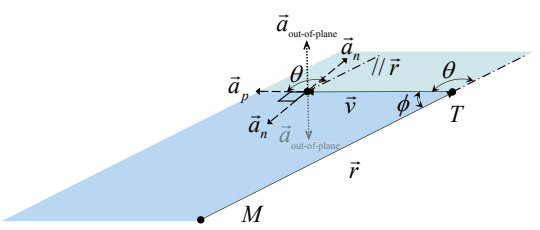

Fig. 5. The Components of $\vec{a}$

The alternative viewpoint from velocity proceeds from the velocity relationship [6]. Let $\vec{v}_{\|}$represent the projection of $\vec{v}$ on $\vec{r}$, thus $\vec{v}_{\perp}=\vec{v}-\vec{v}_{\|}$, as shown in Fig. 4. Based on the triangular equations, we have:

$$
\vec{v}_{\|}=(\vec{v} \cdot \hat{r}) \hat{r} \quad ; \quad \vec{v}_{\perp}=\vec{v}-(\vec{v} \cdot \hat{r}) \hat{r}
$$

Define $\vec{a}_{\perp}=\vec{a}-(\vec{a} \cdot \hat{r}) \hat{r}$ as well as $\vec{v}_{\perp}=\vec{v}-(\vec{v} \cdot \hat{r}) \hat{r}$. Note that they are not in the same direction, because $\vec{r}-\vec{a}$ plane generally does not coincide with $\vec{r}-\vec{v}$ plane. Taking the time derivative of right equation in (35), we have:

$$
\begin{aligned}
\dot{\vec{v}}_{\perp} & =(\vec{a}-(\vec{a} \cdot \hat{r}) \hat{r})-\frac{\vec{v} \cdot \hat{r}}{|\vec{r}|} \vec{v}-\left(\frac{\vec{v} \cdot \vec{v}}{|\vec{r}|}-2 \frac{(\vec{v} \cdot \hat{r})^{2}}{|\vec{r}|}\right) \hat{r} \\
& =\vec{a}_{\perp}-\frac{\vec{v} \cdot \hat{r}}{|\vec{r}|}\left(\vec{v}_{\perp}+(\vec{v} \cdot \hat{r}) \hat{r}\right)-\frac{|\vec{v} \perp+(\vec{v} \cdot \hat{r}) \hat{r}|^{2}}{|\vec{r}|} \hat{r}+2 \frac{(\vec{v} \cdot \hat{r})^{2}}{|\vec{r}|} \hat{r} \\
& =\vec{a}_{\perp}-\frac{\vec{v} \cdot \hat{r}}{|\vec{r}|} \vec{v}_{\perp}-\frac{\left|\vec{v}_{\perp}\right|^{2}}{|\vec{r}|} \hat{r}
\end{aligned}
$$

where the fact $\vec{v}_{\perp} \cdot \hat{r}=0$ has been applied.

\section{B. Guidance Laws Design}

Based on different viewpoints, the 3-D geometric model for intercepting problems is described as (34) and (36). To achieve the interception conditions, controllers of both are designed to assure the stably monotonic decreasing of $\phi$ and $\vec{v}_{\perp}$, respectively.

To deal with the highly nonlinear equation (34), we apply the inverse dynamics strategy. As it is obvious that the last term is less significant than the dominant first term in (34), we let $u=\frac{|\vec{v}|}{|\vec{r}|} \sin \phi-\operatorname{sgn}\left[((\vec{r} \times \vec{v}) \times \vec{v}) \cdot \vec{a}_{n}\right] \frac{\left|\vec{a}_{n}\right|}{|\vec{v}|}$, which implies the control of the simpler plant $\dot{\phi}=u$, and then calculate the control command $\vec{a}_{n}$ from $u$ by inverse dynamics. For the simple plant $\dot{\phi}=u$, we let $\phi=x(t)$ to be scalar state and utilize a modified optimal scheme whose performance index is

$$
J=\frac{s}{2} x_{f}^{2}+\frac{1}{2} \int_{t_{0}}^{t_{f}} e^{-k\left(t_{f}-t\right)} u^{2}(t) d t \quad, s, k>0
$$

Such performance index is specially suitable for the aerodynamically maneuvering missile [4], but this kind of missile is not under our consideration. This performance index also has the merits of more stability and faster convergence. Some detailed discussions of the former can be found in [1, Sec 3.5], whereas the latter can be seen intuitively from the fact that the growing of control cost as time elapses will bring about earlier, faster convergence to the system. These pleasant phenomena are beneficial for solving of intercepting problems, because faster and stabler convergence of state error can suppress the growth of inevitable errors from estimation, modelling, nonlinearity, parasitic dynamics, etc. 
Furthermore, the earlier and faster convergence of state error allows much more control availability in posterior stage of intercepting, which in turn gains much more capability to deal with the final maneuvers of targets. Following the procedure of optimal theory, the optimal control of the above performance index is found as

$$
u=\frac{s k e^{k\left(t_{f}-t\right)}}{s-s e^{k\left(t_{f}-t\right)}-k} x(t) \quad, x=\phi(t)
$$

Once this is done, we can calculate the control commands $\vec{a}_{n}$ from (34) by inverse dynamics:

$$
\operatorname{sgn}\left[((\vec{r} \times \vec{v}) \times \vec{v}) \cdot \vec{a}_{n}\right] \frac{\left|\vec{a}_{n}\right|}{|\vec{v}|}=\frac{|\vec{v}|}{|\vec{r}|} \sin \phi-u
$$

Evaluating the absolute value of both sides, we have the magnitude of $\left|\vec{a}_{n}\right|$ as:

$$
\left|\vec{a}_{n}\right|=|\vec{v}|\left|\frac{|\vec{v}|}{|\vec{r}|} \sin \phi-u\right|
$$

From (39), we also have:

$$
\operatorname{sgn}\left[((\vec{r} \times \vec{v}) \times \vec{v}) \cdot \vec{a}_{n}\right]=\operatorname{sgn}\left(\frac{|\vec{v}|}{|\vec{r}|} \sin \phi-u\right)
$$

Combining it with the fact that $\vec{a}_{n}$ is either in the same or opposite direction of $((\vec{r} \times \vec{v}) \times \vec{v})$, we have:

$$
\frac{(\vec{r} \times \vec{v}) \times \vec{v}}{|(\vec{r} \times \vec{v}) \times \vec{v}|} \cdot \frac{\vec{a}_{n}}{\left|\vec{a}_{n}\right|}=\operatorname{sgn}\left(\frac{|\vec{v}|}{|\vec{r}|} \sin \phi-u\right)
$$

Thereby, we have the direction of $\vec{a}_{n}$ :

$$
\frac{\vec{a}_{n}}{\left|\vec{a}_{n}\right|}=\operatorname{sgn}\left(\frac{|\vec{v}|}{|\vec{r}|} \sin \phi-u\right) \frac{(\vec{r} \times \vec{v}) \times \vec{v}}{|(\vec{r} \times \vec{v}) \times \vec{v}|}
$$

Combining (40) and (43), we finally have:

$$
\vec{a}_{n}=\left(\frac{|\vec{v}|^{2}}{|\vec{r}|} \sin \phi-u|\vec{v}|\right) \frac{(\vec{r} \times \vec{v}) \times \vec{v}}{|(\vec{r} \times \vec{v}) \times \vec{v}|}
$$

If $\vec{a}_{\text {Target }}$ is acquirable, which means $\vec{a}_{T n}$ is known, then the desired perpendicular acceleration commands of missile is $\vec{a}_{M n}=\vec{a}_{T n}-\vec{a}_{n}$. If $\vec{a}_{\text {Target }}$ is merely constant gravity, then $\vec{a}_{M n}=-\vec{a}_{n}$. The main source of accelerating propulsion comes from TVC propellant $N(n t)$ :

$$
\left|\vec{a}_{M}\right|=\frac{N}{m}=\sqrt{\left|\vec{a}_{M n}\right|^{2}+\left|\vec{a}_{M p}\right|^{2}+\left|\vec{a}_{M o}\right|^{2}},
$$

where $m(k g)$ is the total mass of airframe. Knowing the fact from (34) that only the acceleration normal to $\vec{v}$ has effect on the intercepting scenario, we let the complementary part of the constant acceleration $\vec{a}_{M}$ to be along the direction of $\vec{v}$, i.e.:

$$
\vec{a}_{M p}=\frac{\vec{v}}{|\vec{v}|} \sqrt{\left(\frac{N}{m}\right)^{2}-\left|\vec{a}_{M n}\right|^{2}} \quad ; \quad \vec{a}_{M o}=0
$$

Hence, the desired acceleration command of the missile is

$\vec{a}_{d}=\vec{a}_{M n}+\vec{a}_{M p}=\left(\vec{a}_{T n}-\vec{a}_{n}\right)+\frac{\vec{v}}{|\vec{v}|} \sqrt{\left(\frac{N}{m}\right)^{2}-\left|\vec{a}_{T n}-\vec{a}_{n}\right|^{2}}$

which is delivered to the autopilot system.

On the other hand, the same strategy is also applied to (36). We let $u=\vec{a}_{\perp}-\frac{\vec{v} \cdot \hat{r}}{|\vec{r}|} \vec{v}_{\perp}-\frac{\left|\vec{v}_{\perp}\right|^{2}}{|\vec{r}|} \hat{r}$, which implies the control of the simpler plant $\dot{\phi}=u$, and then calculate the control commands from $u$ by inverse dynamics. The last two terms are relatively less than the first term $\vec{a}_{\perp}$ when $\vec{r}$ is large. They will remain less significant than $\vec{a}_{\perp}$ when $\vec{r}$ is decreasing, only if $\vec{v}_{\perp}$ diminishes rapidly as well. This requirement can be satisfied by the same modified optimal scheme used in the derivation of the other viewpoint. For the simple plant $\dot{\vec{v}}_{\perp}=u$, we let $\vec{v}_{\perp}=x(t)$ to be state of $3 \times 1$ vector and utilize the same modified optimal scheme whose performance index is the same as (37). After the same deriving procedure, we have the control command

$$
\vec{a}_{\perp}=\vec{a}_{T \perp}-\vec{a}_{M \perp}=u+\frac{\vec{v} \cdot \hat{r}}{|\vec{r}|} \vec{v}_{\perp}+\frac{\left|\vec{v}_{\perp}\right|^{2}}{|\vec{r}|} \hat{r}
$$

Knowing that $\left|\vec{a}_{M}\right|=\frac{N}{m}, \vec{a}_{M \|}=\left(\vec{a}_{M} \cdot \hat{r}\right) \hat{r}$, and $\vec{a}_{M \perp}=$ $\left(\vec{a}_{M}-\left(\vec{a}_{M} \cdot \hat{r}\right) \hat{r}\right)$, we have

$$
\vec{a}_{M \|}=\hat{r} \sqrt{\left(\frac{N}{m}\right)^{2}-\left|\vec{a}_{M \perp}\right|^{2}}
$$

Hence, the desired acceleration command of the missile is $\vec{a}_{d}=\vec{a}_{M \perp}+\vec{a}_{M \|}=\left(\vec{a}_{T \perp}-\vec{a}_{\perp}\right)+\hat{r} \sqrt{\left(\frac{N}{m}\right)^{2}-\left|\vec{a}_{T \perp}-\vec{a}_{\perp}\right|^{2}}$

which is delivered to the autopilot system.

\section{Integrated Guidance/Autopilot System Analysis}

While deriving the GL, we assume having a perfect autopilot system to execute the guidance commands and thus fulfill the aimed goal. The assumption is unrealistic though, and thus the the stability analysis should be rederived for an integrated G/A system with an imperfect autopilot subsystem. Recalling from (47) and (50) that $\vec{a}_{d}=$ $\vec{a}_{M n}+\vec{a}_{M p}$ and $\vec{a}_{d}=\vec{a}_{M \perp}+\vec{a}_{M \|}$, we simply generalize them as $\vec{a}_{d}=\vec{a}_{d \perp}+\vec{a}_{d \|}$, which are the desired acceleration command given by GL. For an imperfect autopilot system, the actual acceleration output is defined as $\vec{a}_{c}$ rather than $\vec{a}_{d}$, and the relationship between them is found in [7], where the final result is:

$$
\vec{a}_{c \perp}=(1+E) \vec{a}_{d \perp}
$$

If we let $P$ in $S_{a}=\vec{\omega}_{e}+P \vec{q}_{e}$ to be $P=p I_{3 \times 3}$, and if the control $\left|\vec{a}_{d \perp}\right| \neq 0$, we can find the bound of $E$ :

$$
|E| \leq \frac{1}{p} E_{A},
$$

where $E_{A}$ is a bounded positive scalar. Define the Lyapunov function candidate of the integrated G/A system as

$$
V=\frac{1}{2} x^{T} x+\frac{1}{2} S_{a}^{T} J S_{a},
$$

where $x$ equals $\phi$ or $\vec{v}_{\perp}$. Substitute $\vec{a}_{c \perp}$ for $\vec{a}_{d \perp}$ in (34) and (36), it is found [7] that if $|\vec{r}| \neq 0$,

$$
\dot{V} \leq \frac{s k e^{k\left(t_{f}-t\right)}}{s-s e^{k\left(t_{f}-t\right)}-k} x^{T} x\left(1-\frac{E_{A} E_{B}}{p}\right)-\eta_{a}^{T}\left|S_{a}\right|,
$$

where $E_{B}$ is a bounded positive scalar. It can be verified that $\left|\vec{a}_{d \perp}\right|=0$ if the state $x=0$, i.e. $\phi=0$ or $\vec{v}_{\perp}=0$, and that $|\vec{r}|=0$ if the game ends. If we choose a large enough $p>E_{A} E_{B}$, then $\dot{V}$ is N.D. In addition, $V \rightarrow \infty$ as $\left|\left[x^{T} ; S_{a}^{T}\right]\right| \rightarrow \infty$. According to the Lyapunov direct method, we can therefore conclude that with the suggested system parameters the overall integrated G/A system is globally asymptotically stable. 

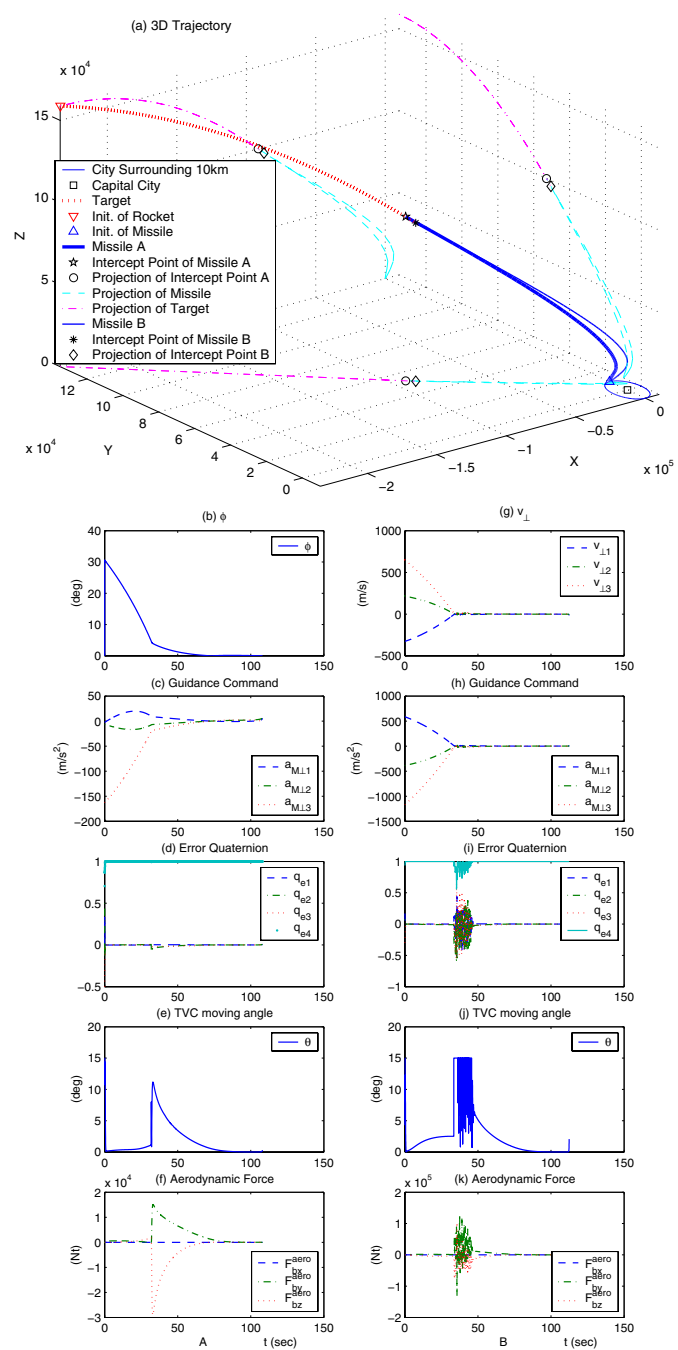

Fig. 6. Missile's Integrated System A (b-f) and B (g-k)

\section{COMPARISONS AND ANALYSIS}

The two proposed missile's integrated G/A systems are compared by simulation in this section. For simplicity, the missile's integrated system with the subsection GL (47) is called A, while with (50) is called B. In this paper, the missile systems are examined for high-tier range, whereas the results for low-tier range can be found in [7]. The missiles travel through the exo- and endo-atmosphere and undergo extremely different environments in the high-tier intercepting scenario, a best way to test the feasibility and performance of the whole-phase-single-strategy controller. In both missile systems, all conditions except for different GLs are set the same: the initial conditions of target, a strategy ballistic missile, is $270 \mathrm{~km}$ far, $158.76 \mathrm{~km}$ high and 5 Mach in horizontal velocity, whereas the defense missile base is settled in $10 \mathrm{~km}$ north of a city with launch velocity $(0,-10,10) \mathrm{m} / \mathrm{s}$. The simplified aerodynamic model from [2] is adopted, and the air density is produced by COESA atmosphere model built-in generator in Matlab.

In Fig. 6(a), the target city of the strategic ballistic missile is drawn, and a circle around the city stands for the field of defense missile base. Besides the 3D trajectories of missile and target, we also have the projections of them on three 2$\mathrm{D}$ planes for clarity. The intercepting time of $\mathrm{A}$ is $108.4 \mathrm{sec}$, and of B is $112.5 \mathrm{sec}$. Both systems seem to achieve the same goal with the nearly equal performance, however, the adjustment of gains in A is easier than B in experimental experience. Besides, it shown in $(c, h)$ that the guidance commands made by $\mathrm{A}$ are much less than by $\mathrm{B}$, and thus so are the requirements of DCS propulsion and the TVC moving angle. The high guidance commands of $\mathrm{B}$ bring about the sudden change of state in (g), and thus cause the chattering phenomenon in $(\mathrm{g})-(\mathrm{k})$. This high control activities, in fact, are originated from the changing of the airframe heading as shown in (a) and thus producing the turning points of the guidance command. In $(\mathrm{e}, \mathrm{j})$ the maximum TVC moving angle $\theta$ of $\mathrm{A}$ is less than $12 \mathrm{deg}$, while of $\mathrm{B}$ reaches the constraint $15 \mathrm{deg}$. In ( $\mathrm{f}, \mathrm{k})$, the aerodynamics produces huge effect inside atmosphere, while affects little outside it in the final intercepting phase.

In summary, the performance of A have some convincingly advantages over $\mathrm{B}$, however, both systems can achieve the same goal with excellent performance, and both have verified the proposed whole-phase-single-strategy missile's integrated G/A system controller designs.

\section{CONCLUSIONS}

Being the decision parts of an missile, GL and autopilot system are discussed is this paper. A wingless airframe based on TVC/DCS and the sliding mode theory is proposed, which makes the autopilot system an whole-phasesingle-strategy robust controller. Besides, two new missile GLs derived from different viewpoints of a geometric model are presented. They both hold the high aiming accuracy, but differ in performance. Finally, the stability of the integrated G/A systems are guaranteed by the suggested system parameters, and verified by various simulations.

\section{ACKNOWLEDGEMENT}

This paper is sponsored by National Science Council under the contract NSC93-2752-E-002-007-PAE.

\section{REFERENCES}

[1] B. D.O. Anderson and J. B. Moore, Optimal Control: Linear Quadratic Methods, Englewood Cliffs, N.J. : Prentice Hall, 1990.

[2] P. Zarchan, Tactical and Strategic Missile Guidance, 4th Edition, Vol. 199, Progress in Astronautics and Aeronautics, AIAA, Inc., 2002.

[3] J. Z. Ben-Asher and I. Yaesh, Advances in Missile Guidance Theory, Vol. 180, Progress in Astronautics and Aeronautics, AIAA, Inc., 1998.

[4] J. Z. Ben-Asher, N. Farber, and S. Levinson, "New Proportional Navigation Law for Ground-to-Air Systems", AIAA Journal of Guidance, Navigation and Dynamics, Vol. 26, No. 5, 2003, pp. 822-825.

[5] C.-Y. Kuo, D. Soeranto, and Y.-C. Chiou, "Geometric Analysis of Flight Control Command for Tactical Missile Guidance", IEEE Trans. on Control Systems Technology, Vol.9, No.2, 2001, pp. 234-243.

[6] Fu-Kuang Yeh, Variable Structure Theory Based Integrated Guidance/Autopilot Design for Maneuverable Flying Vehicles, Ph.D's Dissertation, NTUEE, 2003.

[7] Chia-Hao Cheng, Novel Guidance Law and Autopilot Designs for Intercepting Missiles and Launch Rockets, Master's Thesis, NTUEE, 2004. 